\title{
Retraction Note: Dual loading miR-218 mimics and Temozolomide using AuCOOH@FA-CS drug delivery system: promising targeted antitumor drug delivery system with sequential release functions
}

\author{
$\mathrm{Li} \mathrm{Fan}^{1 \dagger}$, Qian Yang ${ }^{2+}$, Jiali Tan ${ }^{3 \dagger}$, Youbei Qiao', Qiaofeng Wang ${ }^{5}$, Jingya He${ }^{1}$, Hong Wu ${ }^{1 *}$ and Yongsheng Zhang ${ }^{4^{*}}$
}

\section{Retraction Note: J Exp Clin Cancer Res 34, 106 (2015)} https://doi.org/10.1186/s13046-015-0216-8

The Editor-in-Chief has retracted this article. Concerns were raised regarding Figure 2, specifically:

- the top two features in the upper panel appear to be identical to each other, and appear to overlap with the top feature in the lower panel.

- the bottom feature in the upper panel appears to partially overlap with the bottom feature in the lower panel.

- the backgrounds in the upper and lower panel appear to be identical.

The Editor-in-Chief therefore no longer has confidence in the reliability of the data reported in the article.

Li Fan, Quin Yang, Jiali Tan, and Hong Wu agree to this retraction. Youbei Qiao, Qiaofeng Wang, Jingya He, and Yongsheng Zhang have not responded to any correspondence from the editor/publisher about this retraction.

\author{
Author details \\ ${ }^{1}$ Department of Pharmaceutical Analysis, School of Pharmacy, Fourth Military \\ Medical University, Xian 710032, Shaanxi, China. ${ }^{2}$ Institute of Materia Medica, \\ School of Pharmacy, The Fourth Military Medical University, Xian 710032, \\ Shaanxi, China. ${ }^{3}$ Department of Orthodontics, Guanghua School of \\ Stomatology, Hospital of Stomatology, Sun Yat-sen University \& Guangdong \\ Provincial Key Laboratory of Stomatology, Guangzhou 510055, China. \\ ${ }^{4}$ Department of Administrative, Tangdu hosipital, Fourth Military Medical \\ University, Xian 710038, Shaanxi, China. ${ }^{5}$ Department of Pharmaceutical \\ Chemistry, School of Pharmacy Fourth Military Medical University, Xian \\ 710032, Shaanxi, China.
}

Published online: 11 August 2021

* Correspondence: hongwuxa@hotmail.com; zhangys_td@163.com

† Li Fan, Qian Yang and Jiali Tan contributed equally to this work.

'Department of Pharmaceutical Analysis, School of Pharmacy, Fourth Military

Medical University, Xian 710032, Shaanxi, China

${ }^{4}$ Department of Administrative, Tangdu hosipital, Fourth Military Medical

University, Xian 710038, Shaanxi, China

(c) The Author(s). 2021 Open Access This article is licensed under a Creative Commons Attribution 4.0 International License, which permits use, sharing, adaptation, distribution and reproduction in any medium or format, as long as you give appropriate credit to the original author(s) and the source, provide a link to the Creative Commons licence, and indicate if changes were made. The images or other third party material in this article are included in the article's Creative Commons licence, unless indicated otherwise in a credit line to the material. If material is not included in the article's Creative Commons licence and your intended use is not permitted by statutory regulation or exceeds the permitted use, you will need to obtain permission directly from the copyright holder. To view a copy of this licence, visit http://creativecommons.org/licenses/by/4.0/ The Creative Commons Public Domain Dedication waiver (http://creativecommons.org/publicdomain/zero/1.0/) applies to the data made available in this article, unless otherwise stated in a credit line to the data. 